\title{
Comparison of Five TSH-Receptor Antibody Assays in Graves' disease: results from an observational pilot study
}

\author{
Tristan Struja ${ }^{1 * \dagger} \mathbb{D}$, Rebecca Jutzi ${ }^{1 \dagger}$, Noemi Imahorn ${ }^{1 \dagger}$, Marina Kaeslin ${ }^{1}$, Fabienne Boesiger ${ }^{1}$, Alexander Kutz ${ }^{1}$, \\ Esther Mundwiler ${ }^{2}$, Andreas Huber ${ }^{2}$, Marius Kraenzlin ${ }^{4}$, Beat Mueller ${ }^{1,3}$, Christian Meier ${ }^{3,4}$, Luca Bernasconi2 ${ }^{2+}$ and \\ Philipp Schuetz ${ }^{1,3+}$
}

\begin{abstract}
Background: Early diagnosis and relapse prediction in Graves' disease influences treatment. We assessed the abilities of four TSH-receptor antibody tests [TRAb] and one cyclic adenosine monophosphate bioassay to predict relapse of Graves' disease.

Methods: Observational study investigating patients presenting with Graves' disease at a Swiss hospital endocrine referral center or an endocrine outpatient clinic. Main outcomes were diagnosis and relapse of Graves' disease after stop of anti-thyroid drugs. We used Cox regression to study associations of TRAb levels with relapse risk and calculated c-statistics [AUC] to assess discrimination. Blood draws took place as close as possible to treatment initiation.

Results: AUCs ranged from 0.90 (TSAb Biossay by RSR) to 0.97 (IMMULITE TSI by Siemens). Highest sensitivity (94.0\%) was observed for IMMULITE TSI and RSR TRAb Fast, while the greatest specificity (97.9\%) was found with the EliA anti-TSH-R (by Thermo Fisher). In Cox regression analysis comparing the highest versus the lower quartiles, the highest hazard ratio [HR] for relapse was found for BRAHMS TRAK (by Thermo Fisher) (2.98, 95\% Cl 1.13-7.84), IMMULITE TSI (2.40, 95\% Cl 0.91-6.35), EliA anti-TSH-R (2.05, 95\% Cl 0.82-5.10), RSR Fast TRAb (1.80, 95\% Cl 0.73-4.43), followed by RSR STIMULATION (1.18, 95\% Cl $0.46-2.99$ ). Discrimination analyses showed respective AUCs of $0.68,0.65,0.64,0.64$, and 0.59 .
\end{abstract}

Conclusion: The assays tested had good diagnostic power and relapse risk prediction with few differences among the new assays. Due to the small sample size and retrospective design with possible selection bias, our data need prospective validation.

Keywords: Thyroid, Graves' disease, TRAb, Bioassay

\section{Introduction}

Graves' disease [GD] is among the leading causes of hyperthyroidism affecting approximately $0.5 \%$ of the general population, especially young women [1]. It is caused by the presence of autoantibodies to the thyrotropin [TSH] receptor leading to unregulated production and secretion of thyroid hormones [1]. Typically, GD is characterized by suppressed serum TSH and overproduction of thyroid hormones thyroxine and triiodothyronine [T4 and

\footnotetext{
* Correspondence: tristan.struja@gmail.com

'Tristan Struja, Rebecca Jutzi and Noemi Imahorn equally contributing first authors

†Luca Bernasconi and Philipp Schuetz equally contributing senior authors

${ }^{1}$ Medical University Department, Clinic for Endocrinology, Diabetes \&

Metabolism, Kantonsspital Aarau, Tellstrasse, CH-5001 Aarau, Switzerland

Full list of author information is available at the end of the article
}

T3] [2]. To distinguish GD from other causes of hyperthyroidism, measurement of TSH-receptor autoantibodies [TRAb] is usually helpful [3].

Most patients are initially treated with antithyroid drugs [ATD] for a recommended duration of 12 to 18 months, but this therapy may have adverse effects such as agranulocytosis, rash, joint pain, and hepatitis [4]. Other treatment options like radioactive iodine or total thyroidectomy are preferred in patients with relapse after ATD, however, these treatments usually lead to persisting hypothyroidism and lifelong T4-replacement $[1,2]$. Importantly, the rate of relapse after ATD is high (around 50\%) [1]. To predict relapse in GD, the Graves' Recurrent Events After Therapy [GREAT] score has been proposed and recently validated

(c) The Author(s). 2019 Open Access This article is distributed under the terms of the Creative Commons Attribution 4.0 International License (http://creativecommons.org/licenses/by/4.0/), which permits unrestricted use, distribution, and reproduction in any medium, provided you give appropriate credit to the original author(s) and the source, provide a link to the Creative Commons license, and indicate if changes were made. The Creative Commons Public Domain Dedication waiver (http://creativecommons.org/publicdomain/zero/1.0/) applies to the data made available in this article, unless otherwise stated. 
by our research group $[5,6]$. This score is based on clinical and biochemical parameters. Age at diagnosis ( $\geq 40$ years), higher serum fT4 ( $\geq 40 \mathrm{pmol} / \mathrm{L})$, higher serum TRAb $(\geq 6$ $\mathrm{U} / \mathrm{L}$ ), and larger goiter sizes (WHO class II-III) were associated with higher recurrence rates. In the original study, the GREAT score discriminated patients with relapse from those without relapse with a fair prognostic accuracy area under the curve [AUC] of 0.67 (95\% confidence interval [CI]: 0.54-0.77). There was a $68 \%$ risk of relapse in patients with class III (4-6 points in the GREAT score) compared to $16 \%$ in patients with class I ( $0-1$ points in the GREAT score) and $44 \%$ in patients with class II (2-3 points in the GREAT score) [5]. In addition to this clinical score, pooled evidence from a systematic review and meta-analysis showed that elevated first to third generation assay TRAb levels at diagnosis are associated with higher relapse rates [7].

Recently, two new fully automated TRAb immunoassays have become available, IMMULITE TSI (Siemens Healthineers) and EliA anti-TSH-R (Thermo Fisher Scientific). The former uses recombinant thyrotropin receptor chimeras and is based on a bridge technology. The latter is based on immunological competitive reactions between patient's autoantibodies and human monoclonal antibodies for the binding to human recombinant TSH receptors, similarly to BRAHMS TRAK and RSR Fast TRAb. Their sensitivity and specificity in the diagnosis of GD have been described to be high and comparable to other 3rd generation TRAb tests $[8,9]$. The advantage of these new immunoassays is not only their automated routine, but in particular for the IMMULITE TSI its declared ability to specifically detect only TSH-receptor stimulatory antibodies. This property has been so far reserved to laborious bioassays $[8,10]$. Yet, the utility of these new third-generation immunoassays in predicting GD relapse at diagnosis has not been assessed so far. Herein, we compared five different TSH-receptor antibody tests for their ability to diagnose and predict relapse of Graves' disease.

\section{Methods}

In this 10-year retrospective, observational cohort study we analyzed data from 332 patients from a large endocrine outpatient clinic and one hospital based endocrine tertiary referral center in Switzerland. The primary outcome of this study was relapse in GD after an ATD treatment cycle similar to a previous study [6]. Patients were treated with ATD in a titration regimen upon their first episode of hyperthyroidism. Inclusion criteria were a first episode of GD defined as suppressed serum TSH $(<0.01 \mathrm{mU} / \mathrm{l})$, elevated $\mathrm{fT} 4$, and if available, diffuse increased uptake in thyroid scintigraphy ( $99 \mathrm{~m}$-Tc-pertechnetate). Patients with follow-up after start of ATD treatment $<24$ months, ATD treatment $<12$ months, initial ablative therapy (i.e. surgery or RAI), and time gap between initiation of treatment and blood draw over 2.5 months were excluded. This left 83 GD patients for analysis. In the diseased control group, we included 48 patients with Hashimoto's thyroiditis $(n=16)$, thyroid autonomy $(n=13)$, thyroiditis $(n=9)$, and other hyperthyroid-associated diseases $(n=10$, i.e. toxic goiter, amiodarone-induced thyroiditis). Relapse had to be established by suppressed TSH and elevated peripheral hormone (fT4).

\section{Clinical data}

We collected clinical data by medical charts and electronic records review and if necessary, we complemented missing follow-up data by phone calls to patients and general practitioners. We gathered the following clinical parameters from the first patient's visit: goiter size (WHO classification, 0-III); thyroid volume assessed by sonography; date of first ATD and the type of drug used; smoking status (yes or no); presence of Graves' orbitopathy (yes or no); anti-thyroperoxidase-antibodies [TPO-Ab]; TRAb levels; and whether any other autoimmune diseases were present. During the course of disease, we observed TSH levels in constant intervals, date of ATD withdrawal, changes in drug regimen and reasons therefore (i.e. adverse effects), date of relapse, and, if no relapse occurred, date of last consultation. All patients were usually treated for 12 to 18 months with carbimazole or propylthyouracil using a titration regime.

\section{Laboratory measurements}

After blood withdrawal, samples were directly centrifuged and analyzed for serum TSH, fT4, anti-TPO-Ab, and TRAb levels by commercially available laboratory assays as part of the clinical routine in the different participating sites. Routine TRAb were either measured at the Kantonsspital Aarau or at Hormony (specialized laboratory on hormone analysis, Prof. J. Girard, Basel, Switzerland). The TRAb assays routinely used and their technical specifications are listed in Additional file 1: Table S1.

Leftover serum aliquots were stored at $-20^{\circ}$ Celsius and mean duration storage time was 46 months (median 46 months; 17 to 70 months interquartile range). TRAb concentration was measured with the following assays according to the manufacturers' instructions: BRAHMS TRAK human KRYPTOR (Thermo Fisher Scientific, Germany), IMMULITE 2000 TSI (Siemens, Healthineers, Germany), EliA anti-TSH-R (Thermo Fisher Scientific, Germany), and ELISA RSR TRAb Fast (RSR Limited, UK). Cut-offs suggested by the manufacturers were used to evaluate diagnostic performance. For the detection of stimulating type (TSAb) and blocking type (TSBAb) autoantibodies patient sera were shipped on dry ice to RSR Limited (UK) who performed CHO-cell based, cAMP-dependent bioassays with

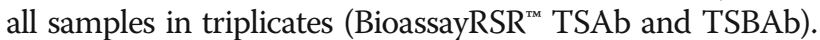
Intracellular cAMP was subsequently determined using the 
Direct Cyclic AMP ELISA (Enzo Life Sciences, Switzerland). A stimulation of $\geq 150 \%$ compared to the healthy blood donor control was considered as a positive result for a stimulating activity. Blocking activity was present if $\geq 30 \%$ inhibition of TSH stimulation compared to the healthy blood donor control was observed. For more information on the assays tested, please refer to the appropriate section of the Additional file 1.

\section{Statistical analysis}

We recently externally validated the GREAT score. Primary objective of our study was to compare the capability of the different TRAb assays in diagnosing GD and to analyze whether the GREAT score could be further improved by the addition of novel and more specific TRAb assays. For this purpose, we performed similar statistical analyses as described before [5]. In brief, we used univariate and multivariate Cox-proportional hazard regression models to study the association of previously suggested risk factors separately and combined in the GREAT score with the risk for time to relapse. For dichotomization of variables, we used the same cut-offs as in the original report, except for the new assays where separated data into four quantiles. We also calculated the GREAT score risk classes as suggested [5]. Kaplan-Meier method was used to graphically display data with use of the log-rank test. Area under the receiver operator curve [AUC] [ROC] over the whole follow up time after ATD stop was used to assess discriminative power of the GREAT score.

All significance tests were two-sided and $P<0.05$ was considered statistically significant. Categorical variables are expressed as percentages (counts) and continuous variables as mean and standard deviation. If applicable, a 95\% $\mathrm{CI}$ is provided. As our not normally distributed data was right-skewed, we log transformed (base 10) it before analysis. Survival analysis and ROC curves for relapse were conducted using Stata software version 12.1 (Stata Corp., College Station, TX, USA). Diagnostic performance of the different TRAb assays was analyzed using MedCalc Statistical Software version 15.11.4 (MedCalc Software bvba, Ostend, Belgium; https://www.medcalc.org; 2015).

\section{Results}

\section{Baseline characteristics}

We included 131 patients in this cohort (14.5\% males). Out of the GD subpopulation, $18(21.7 \%)$ had a relapse after a median follow-up time of $22(9 ; 33$ interquartile range [IQR]) months after ATD start, or 17 (7; 32 IQR) months after ATD withdrawal, respectively. To illustrate patient inclusion, we integrated a selection flow sheet into the appendix (see Additional file 1: Figure S1). Tables 1 and 2 shows details of the patient population stratified by relapse and diagnosis. Patients with relapse had a higher rate of endocrine orbitopathy, higher $\mathrm{fT}_{4}, \mathrm{TPO}-\mathrm{Ab}$, and $\mathrm{TRAb}$ levels. After relapse, all but two patients (they opted for surgery) chose to continue ATD treatment.

A total of $7.3 \%$ of the patients were initially treated with propylthyouracil, whereas the remainder received carbimazole. Ten patients were switched from carbimazole to propylthyouracil or vice versa. Most changes occurred because of pregnancies $(n=1)$ or skin rashes $(n=7)$. Besides one case of hepatitis (carbimazole group), no serious adverse effects occurred. Especially, there was no case of liver failure, agranulocytosis or death.

\section{Diagnostic performance}

The distribution of TRAb levels of the 83 GD patients and 48 diseased controls measured by the different assays is depicted in Fig. 1. ROC curve analysis revealed AUCs ranging from 0.90 (TSAb Biossay - RSR Limited) to 0.97 (IMMULITE TSI - Siemens) (Table 3). Highest sensitivity (94.0\%) was observed for IMMULITE TSI (Siemens) and RSR TRAb Fast (RSR Limited) assays while the greatest specificity (97.9\%) was found with the EliA anti-TSH-R (Thermo Fisher Scientific). Figure 2 shows the distribution of TRAb concentrations by diagnosis.

\section{Discrimination statistics for relapse assessment}

Figure 3 shows distribution of TRAb levels of the 83 GD patients depicted. Median and IQR values according to the figure are presented in the first two columns of Tables 1 and 2 . We calculated the AUCs to assess discrimination of assays in regard to prediction of relapse (see Additional file 1: Figure S2). AUC figures for the GREAT score were recalculated for our present cohort according to our initial publication (see Table 3) [6]. Most assays predicted the outcome relapse with moderate AUCs of around 0.67 to 0.71 . Combined with the GREAT score, they did not show a significantly improved predictive ability. All assays performed in a similar range except for the bioassay.

\section{Cox proportional hazard regression analysis}

To analyze whether the TRAb assays further improve the predictive ability of the GREAT score, we modeled a univariate and a multivariate cox regression analysis. The results from the TRAb assays were split according to their quartiles and we compared the highest versus the remaining three quartiles (see Table 4). In univariate analysis, we modeled the TRAb level against time to relapse after ATD withdrawal. All assays showed significant associations but with very wide CI due to the small sample size. Incorporation of the TRAb assay results into a multivariate model (i.e. the existing GREAT score without the routine TRAb) provided improved hazard ratios with the BRAHMS assay as compared to the GREAT score with the routine TRAb. Whereas IMMULITE, EliA anti-TSH-R, and RSR TRAb Fast only improved the 
Table 1 Baseline characteristics of GD patients

\begin{tabular}{|c|c|c|c|}
\hline Numbers (\%) & GD no relapse & GD relapse & $P$ - value \\
\hline Sex & $65(49.6 \%)$ & $18(13.7 \%)$ & \\
\hline Female & $55(85 \%)$ & $15(83 \%)$ & 0.89 \\
\hline Male & $10(15 \%)$ & $3(17 \%)$ & \\
\hline Age (years), mean $\pm \mathrm{SD}$ & $52 \pm 13$ & $47 \pm 13$ & 0.13 \\
\hline $\mathrm{BMI}\left(\mathrm{kg} / \mathrm{m}^{2}\right)$, mean $\pm \mathrm{SD}$ & $24 \pm 4.6$ & $25 \pm 3.7$ & 0.23 \\
\hline Smokers & $9(25 \%)$ & $1(20 \%)$ & 0.81 \\
\hline Treatment duration (months), median (IQR) & $19(18,21)$ & $18(17,21)$ & 0.81 \\
\hline Follow up duration after ATD stop (months), median (IQR) & $11(3,36)$ & $1(0.5,11)$ & $<0.01$ \\
\hline Thyroid volume (ml), median (IQR) & $14(11,18)$ & $14(9.6,16)$ & 0.71 \\
\hline \multicolumn{4}{|l|}{ Goiter size (WHO grade, 0-III) } \\
\hline 0 & $33(62 \%)$ & $10(67 \%)$ & \multirow[t]{4}{*}{0.84} \\
\hline । & $12(23 \%)$ & $4(27 \%)$ & \\
\hline$\|$ & $7(13 \%)$ & $1(7 \%)$ & \\
\hline III & $1(2 \%)$ & $0(0 \%)$ & \\
\hline Endocrine orbitopathy (N/\%) & $18(28 \%)$ & 7 (39\%) & 0.36 \\
\hline fT4 (pM), median (IQR) & $30(21,36)$ & $35(20,55)$ & 0.31 \\
\hline $\mathrm{T} 3(\mathrm{pM})$, median (IQR) & $3.5(2.5,4.4)$ & $2.9(2.5,6.4)$ & 0.90 \\
\hline fT3 $(\mathrm{pM})$, median (IQR) & $10(7.7,17)$ & $21(14,29)$ & 0.06 \\
\hline TPO-Ab (U/I), median (IQR) & $89(49,475)$ & $120(90,357)$ & 0.49 \\
\hline Routine TRAb assay ${ }^{\mathrm{a}}(\mathrm{U} / \mathrm{L})$, median (IQR) & $5.4(2.8,10)$ & $12(3.5,27)$ & 0.10 \\
\hline IMMULITE TSI (Cut-off $0.55 \mathrm{U} / \mathrm{L})$, median (IQR) & $3.4(1.6,7.5)$ & $5.6(3.6,17)$ & 0.04 \\
\hline BRAHMS TRAK (Cut-off $1.8 \mathrm{U} / \mathrm{L}$ ), median (IQR) & $4.6(2.6,11)$ & $8.6(5.1,20)$ & 0.02 \\
\hline EliA anti-TSH-R (Cut-off $2.9 \mathrm{U} / \mathrm{L})$, median (IQR) & $4.4(2.9,9.6)$ & $7.4(4.4,13)$ & 0.04 \\
\hline RSR TRAb Fast (Cut-off $1.0 \mathrm{U} / \mathrm{L})$, median (IQR) & $4.3(2.7,7.5)$ & $6.9(4.1,16)$ & 0.06 \\
\hline RSR-bioassay STIMULATION (Cut-off 150\%), median (IQR) & $461(192,835)$ & $536(291,1419)$ & 0.26 \\
\hline Additional autoimmune disease: & & & 0.51 \\
\hline GIT (IBD, CD, pernicious anemia) & 1 & 1 & \\
\hline T1DM & 1 & 0 & \\
\hline Other & 1 & 0 & \\
\hline
\end{tabular}

ariginally, study centers used different commercially available assays with different cut-offs, for details please see Additional file 1: Table S1

$\S$ categorical and binary variables were compared by Pearson's chi-squared test, continuous, non-normally distributed variables were compared by Wilcoxon rank-

sum test; $P$-values not adjusted to multiple testing

GREAT score for GREAT class II, but not class III. To illustrate these findings, we plotted Kaplan-Meier survival curves (see Fig. 4 and Additional file 1: Figure S3). Also, we further added either smoking or orbitopathy as covariates into the model. There were no significant changes in HRs (results not shown).

\section{Discussion}

Based on this observational, secondary analysis of blood samples collected in a representative patient population from a multicenter study, we tested five TRAb assays for their power to diagnose and predict relapse in GD patients. Three competition assays, including the recently released automated EliA anti-TSH-R, an automated assay based on bridge technology [9], and one cell-based bioassay have been considered.

\section{Diagnosis of GD}

ROC curve analysis demonstrated highly comparable AUCs for the different assays except for the bioassay which showed a fairly lower AUC. Sensitivities varied from $79.5 \%$ (EliA anti-TSH-R) to $94.0 \%$ (IMMULITE TSI and RSR TRAb Fast). Previous studies described slightly higher sensitivities for IMMULITE TSI between 95 and $100 \%$ [8, 10-12], while the manufacturer suggested a sensitivity for EliA anti-TSH-R varying between $83 \%$ at a cut-off $2.9 \mathrm{U} / \mathrm{l}$ and $79 \%$ at $3.3 \mathrm{U} / \mathrm{l}$ (grey-zone 2.9-3.3 U/l) [13]. Thus, in our study, the performance of the bioassay was inferior to that reported in former 
Table 2 Baseline characteristics of the non-GD patients

\begin{tabular}{|c|c|c|c|c|c|}
\hline Numbers (\%) & Hashimoto's Thyroiditis & Thyroiditis & Toxic nodular goiter & Other $^{a}$ & $P$-value \\
\hline Sex & $16(12.2 \%)$ & $9(6.9 \%)$ & $13(9.9 \%)$ & $10(7.6 \%)$ & \\
\hline Female & $16(100.0 \%)$ & $8(89 \%)$ & $12(92 \%)$ & $6(60.0 \%)$ & 0.23 \\
\hline Male & $0(0.0 \%)$ & $1(1 \%)$ & $1(8.0 \%)$ & $4(40.0 \%)$ & \\
\hline Age (years), mean $\pm \mathrm{SD}$ & $52 \pm 13$ & $51 \pm 15$ & $68 \pm 16$ & $53 \pm 22$ & 0.04 \\
\hline $\mathrm{BMI}\left(\mathrm{kg} / \mathrm{m}^{2}\right)$, mean $\pm \mathrm{SD}$ & $25 \pm 3.7$ & $25 \pm 3.2$ & $24 \pm 3.3$ & $23 \pm 3.7$ & 0.68 \\
\hline IMMULITE TSI (Cut-off 0.55 U/L), median (IQR) & $0.05(0.05,0.09)$ & $0.05(0.05,0.2)$ & $0.05(0.05,0.05)$ & $0.05(0.05,0.05)$ & 0.20 \\
\hline BRAHMS TRAK (Cut-off $1.8 \mathrm{U} / \mathrm{L})$, median (IQR) & $0.2(0.2,0.2)$ & $0.2(0.2,0.2)$ & $0.2(0.2,0.62)$ & $0.2(0.2,0.2)$ & 0.88 \\
\hline EliA anti-TSH-R (Cut-off $2.9 \mathrm{U} / \mathrm{L})$, median (IQR) & $1.4(1.4,1.4)$ & $1.4(.9,1.9)$ & $1.4(.9,1.4)$ & $1.4(1.2,1.7)$ & 0.60 \\
\hline RSR TRAb Fast (Cut-off $1.0 \mathrm{U} / \mathrm{L})$, median (IQR) & $0.9(0.9,0.9)$ & $0.9(0.9,1.1)$ & $0.9(0.9,0.9)$ & $0.9(0.9,1.1)$ & 0.09 \\
\hline RSR-bioassay STIMULATION (Cut-off 150\%), median (IQR) & $94(85,119)$ & $98(91,131)$ & $87(86,92)$ & $98(93,182)$ & 0.12 \\
\hline
\end{tabular}

Abbreviation: $C D$ celiac disease, GIT gastrointestinal tract, IBD inflammatory bowel disease, IQR interquartile range, $p M$ pmol/L, SD standard deviation, T1DM type 1 diabetes mellitus

${ }^{a}$ Other includes: amiodarone induced hyperthyroidism, euthyroid sick syndrome, postpartum thyroiditis, silent thyroiditis, euthyroid goiter, follicular and papillary carcinoma, functional TSH suppression after i.v. contrast agent

${ }^{\S}$ categorical and binary variables were compared by Pearson's chi-squared test, continuous, non-normally distributed variables were compared by Wilcoxon ranksum test; $P$-values not adjusted to multiple testing

studies examining different bioassay systems [12, 14, 15]. BRAHMS TRAK showed a higher sensitivity than previously reported by Diana et al. [12]. The RSR TRAb Fast, a modified version of the RSR 3rd generation TRAb ELISA [16], exhibited a sensitivity of $94 \%$ which is higher than the $85-93 \%$ observed with the unmodified assay [17, 18]. Overall, we report lower sensitivities compared to those described in a meta-analysis performed by Tozzoli et al. [19] examining different 3rd generation assays (pooled sensitivity of $97.4 \%$ ). There are several explanations for these differences. First, we evaluated a rather small cohort of patients and due to the retrospective design, selection bias towards lower severity patients is likely. This also explains to lower risk for relapse in our cohort as compared to previous studies [20]. Still, out of the GD 268 patients with blood samples (see Additional file 1: Figure S1) 25 and 26 received surgery or RAI in the long term, respectively and median time to definitive therapy after diagnosis was 35 months (median, IQR 8-71, mean 47 months) which argues against selection bias. Second, previous studies compared assay performance between GD patient and healthy volunteers, while we included patients with different types of thyroid pathologies. Thus, our results may better reflect real life indications for TRAb.

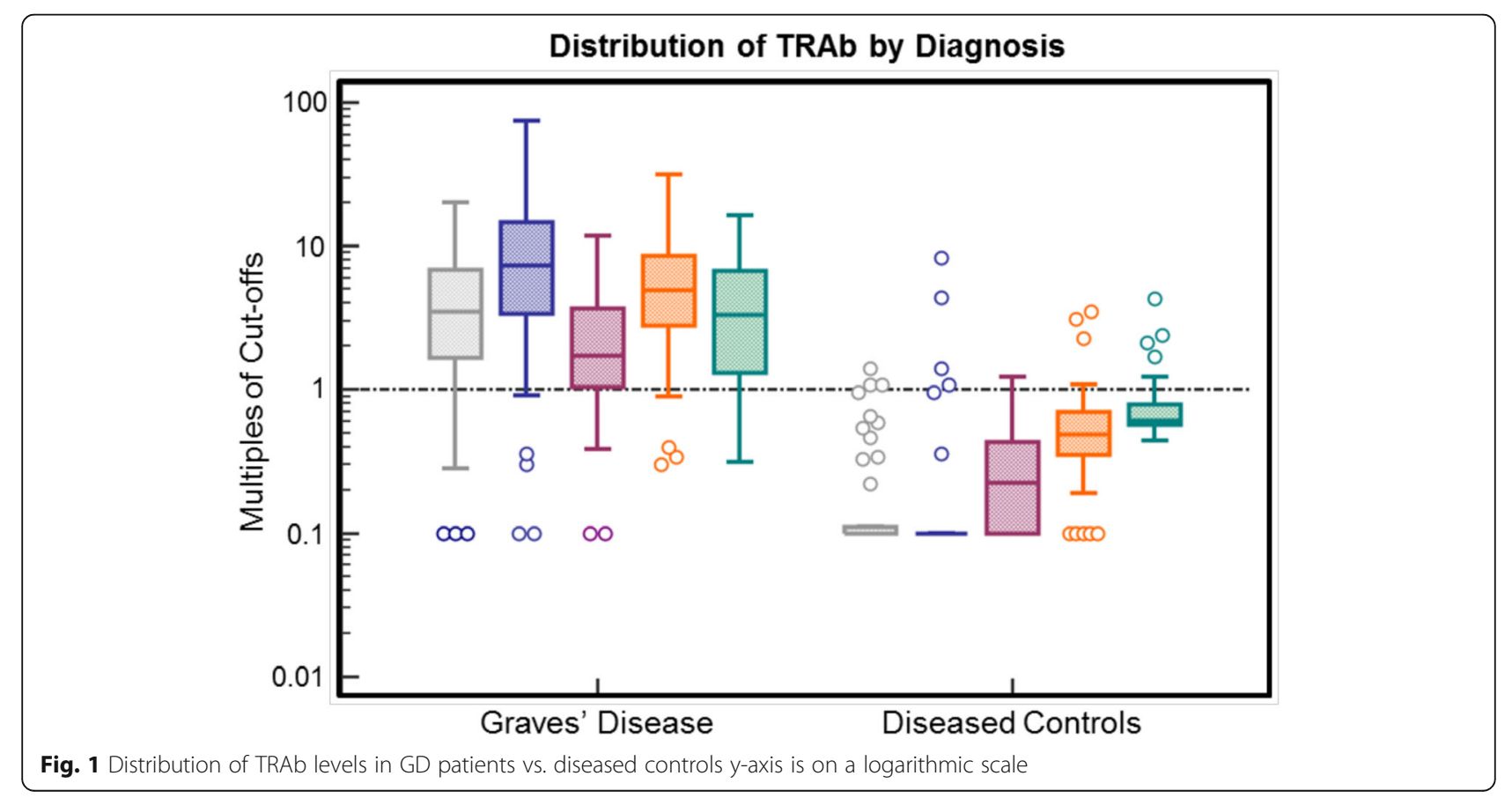


Table 3 AUC for GD diagnosis and relapse compared to GREAT score and refitted with new TRAb's ${ }^{b}$

\begin{tabular}{|c|c|c|c|c|c|c|c|c|}
\hline \multirow[b]{2}{*}{ Discriminator } & \multicolumn{4}{|l|}{ DIAGNOSIS } & \multicolumn{4}{|c|}{ GD RELAPSE PREDICTION } \\
\hline & Sensitivity [\%] & Specificity [\%] & AUC & $95 \% \mathrm{Cl}$ & AUC & $95 \% \mathrm{Cl}$ & Improved AUC & $95 \% \mathrm{Cl}$ \\
\hline GREAT score without routine TRAb & & & & & 0.57 & $(0.43-0.71)$ & & \\
\hline \multirow[t]{2}{*}{ GREAT score with routine TRAb ${ }^{a}$} & & & & & 0.69 & $(0.56-0.81)$ & & \\
\hline & & & & & & & \multicolumn{2}{|c|}{ GREAT score with new assay } \\
\hline BRAHMS TRAK & 86.7 & 93.7 & 0.96 & $(0.91-0.99)$ & 0.71 & $(0.57-0.86)$ & 0.67 & $(0.53-0.81)$ \\
\hline IMMULITE TSI & 94.0 & 91.7 & 0.97 & $(0.92-0.99)$ & 0.69 & $(0.54-0.84)$ & 0.66 & $(0.53-0.79)$ \\
\hline \multicolumn{9}{|l|}{ EliA anti-TSH-R } \\
\hline$\geq 2.9 \mathrm{U} / \mathrm{L}$ & 79.5 & 93.7 & 0.95 & $(0.90-0.98)$ & 0.68 & $(0.52-0.83)$ & 0.68 & $(0.54-0.82)$ \\
\hline$>3.3 \mathrm{U} / \mathrm{L}$ & 71.1 & 97.9 & 0.95 & $(0.90-0.98)$ & & & & \\
\hline RSR TRAb Fast & 94.0 & 89.6 & 0.96 & $(0.91-0.99)$ & 0.67 & $(0.50-0.83)$ & 0.64 & $(0.50-0.78)$ \\
\hline RSR-bioassay STIMULATION & 81.9 & 87.5 & 0.90 & $(0.84-0.95)$ & 0.62 & $(0.45-0.78)$ & 0.62 & $(0.48-0.76)$ \\
\hline
\end{tabular}

It is well known that TRAb levels decline gradually under ATD treatment until they disappear in about three quarters of the patients after 18 months [21]. In our opinion, this has a limited influence on our results as we only included patients up to 2.5 months after ATD initiation. By definition, every untreated GD patient should have TRAb. However, in the past up to 6-7\% of GD patients were described to lack detectable TRAb, albeit these numbers are rather based on earlier TRAb assay generations $[22,23]$. Nevertheless, in our study four sera of GD patients $(4.8 \%)$ were negative with all assays.

Specificities ranged from $87.5 \%$ for the bioassay to $97.9 \%$ for the EliA anti-TSH-R at the upper cut-off $(3.3 \mathrm{U} / \mathrm{l})$. This is in agreement with the specificity of $97.7 \%$ published by Luther et al. [13] for the EliA anti-TSH-R. With EliA anti-TSH-R only one patient of the control group (autoimmune thyroiditis) had a borderline result (MOC 1.03 at cut-off 3.3). This serum was positive with all other assays (MOCs: RSR Fast TRAb 3.1, IMMULITE TSI 4.36, BRAHMS TRAK 1.39, TSAb Bioassay 1.29). Previously published specificities are generally higher (98.7-100\%) compared to our results $[8,10,11,17,19]$. However, many studies included healthy subjects, whereas our control group consisted solely of thyroid-related disease patients. The frequency of TRAb positivity for multinodular toxic goiter or primary autoimmune hypothyroidism has been shown to be about 10\% with RSR 3rd generation TRAb ELISA [17] and $10 \%$ for Hashimoto's thyroiditis (HT) with BRAHMS TRAK [12]. According to the literature, stimulating TRAb can be found in $5.5-22 \%$ of HT patients $[24,25]$. TRAb were detected in 1 out of 15 patients (6.7\%) in the HT-control group. This particular serum was positive with all binding assays (MOCs: RSR Fast TRAb 2.28, IMMULITE TSI 1.39, BRAHMS TRAK 1.08) except with EliA anti-TSH-R (MOC 0.79 at cut-off 2.9) and TSAb bioassay (MOC 0.57). In this case both TSAb and TBAb bioassays were negative. According to Diana et al. TBAb can be observed in $4.2 \%$ of GD and in $9.3 \%$ of HT patients [26]. In our study, TBAb were detected in low amount in only one patient with silent thyroiditis (data not shown). This could be due to the different bioassay setup used in the study by Diana et al. [26] or to the limited sample size of our retrospective analysis.

\section{Prediction of relapse}

Added to the GREAT score two assays (i.e. BRAHMS TRAK, and IMMULITE TSI) showed a statistically significant improvement of its predictive capabilities. Thus, these assays might provide a clinical benefit in predicting the relapse risk of newly diagnosed GD patients offered ATD therapy.

Somewhat surprising was the finding that concentrations of EliA anti-TSH-R did not seem to differ largely between the two groups (see Tables 1 and 2 for medians and Fig. 3 for box-plots), whereas the average HR for relapse prediction for the assay itself was the highest of all (see column "HR for assay alone (Q4 vs. Q1-3) (95\% CI)" in Table 4). We think that this finding occurred by chance due to our small sample size as suggested by the wide confidence intervals. In this subsample of our previously published dataset [6], we observed a rather low overall recurrence rate of only $21.7 \%$ (originally $50.1 \%$ ). This is slightly lower than usually reported from other cohorts in the past (30-60\%) [9-11]. Although we had such a low incidence of events, we still observed statistically significant findings. Thus, we are confident that our data are robust and valid. Especially, as we ensured a high follow up rate in our original study by performing follow-up interviews with patients and/or their primary care physicians in case there had not been a contact within the last 6 months with 

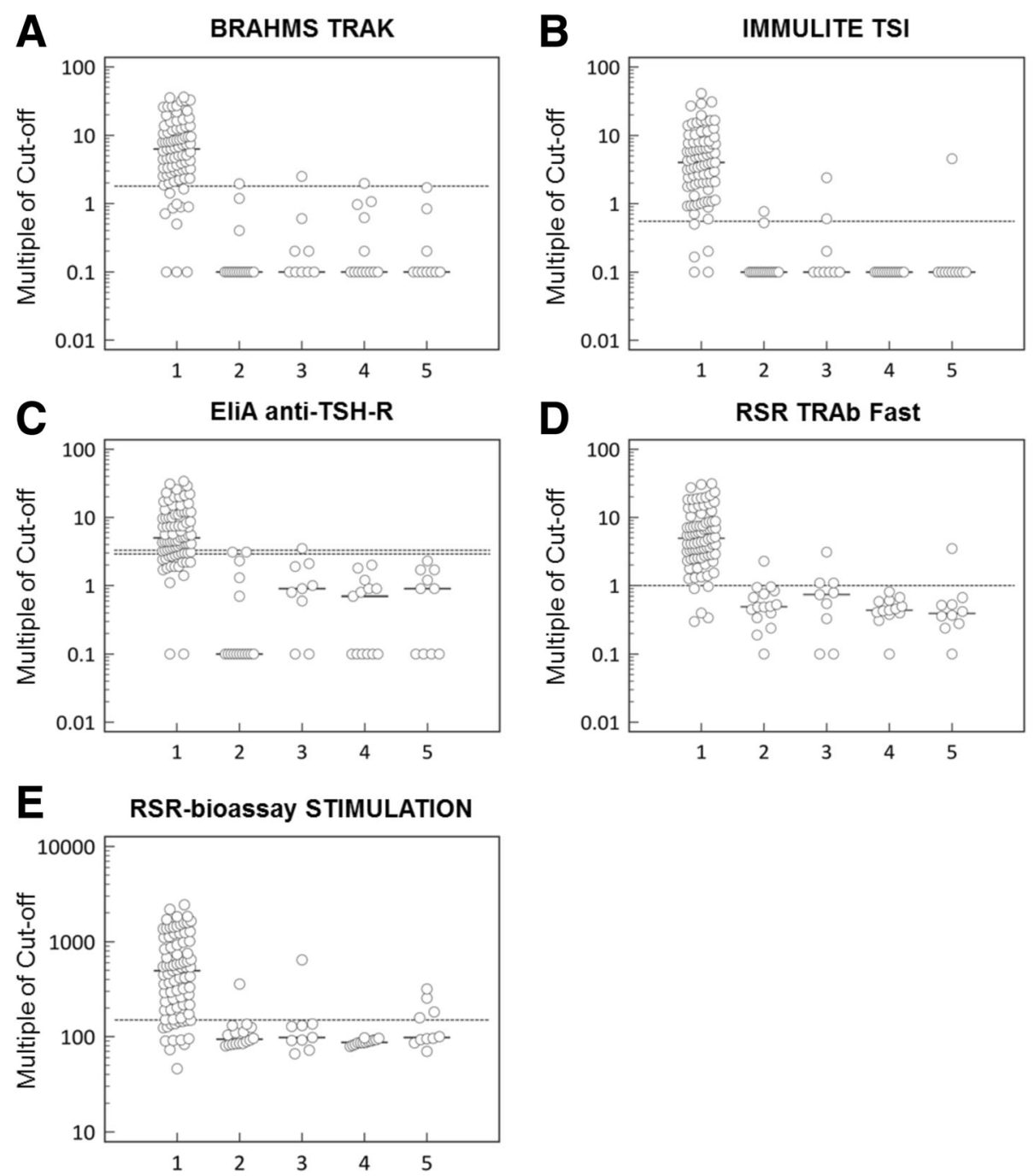

Fig. 2 Distribution of TRAb levels by diagnosis y-axes are on a logarithmic scale. 1, Graves' disease. 2, Hashimoto's thyroiditis. 3, Thyroiditis. 4, Toxic nodular goiter. 5, Other (i.e. amiodarone induced hyperthyroidism, euthyroid sick syndrome, postpartum thyroiditis,silent thyroiditis, euthyroid goiter, follicular and papillary carcinoma, functional TSH suppression after i.v. contrast agent). Panel a TRAb from Brahms. Panel b TRAb from Siemens. Panel c TRAb from Thermo Fisher Scientific. Panel d TRAb from RSR Limited. Panel e TSAb from RSR Limited

a study center. In Switzerland, patients typically stay with their general practitioner for many years.

The overall predictive accuracy of the TRAb assays alone is ranging from 0.67 to 0.71 , being like the GREAT score with the routine TRAb (AUC of 0.69). Although some new TRAb assays showed statistically significant improvements, it is less clear if these improvements prove clinically relevant.

Fitted into a survival model, we compared the fourth quartile of TRAb assay results against the remaining lower three. HR for all TRAb assays were in the same range as those for the GREAT class II (i.e. HR 1.79; 95\% CI 1.42-2.27). When added to the GREAT score predictive ability improved even further. Hence, we believe that the TRAb assays used in our study provide some benefit for patient assessment with only slight differences between the different manufacturers. There is a slight reduction in hazard ratios in GREAT class III, which we attribute mainly to the variance caused by few data points in this group.

All these findings do not apply to the cAMP bioassay. Although disease course prediction has been reported to be improved by using bioassays, we could not replicate similar results $[27,28]$. Even the IMMULITE TSI assay by Siemens did not have unrivalled predictive capabilities, albeit it is supposed to specifically detect only stimulatory antibodies. One reason might be that our sample size has not been large enough for a confirmatory finding.

Overall, the fact that a single factor in predicting the outcome of GD patients under ATD therapy is insufficient and needs to be combined with other factors. 


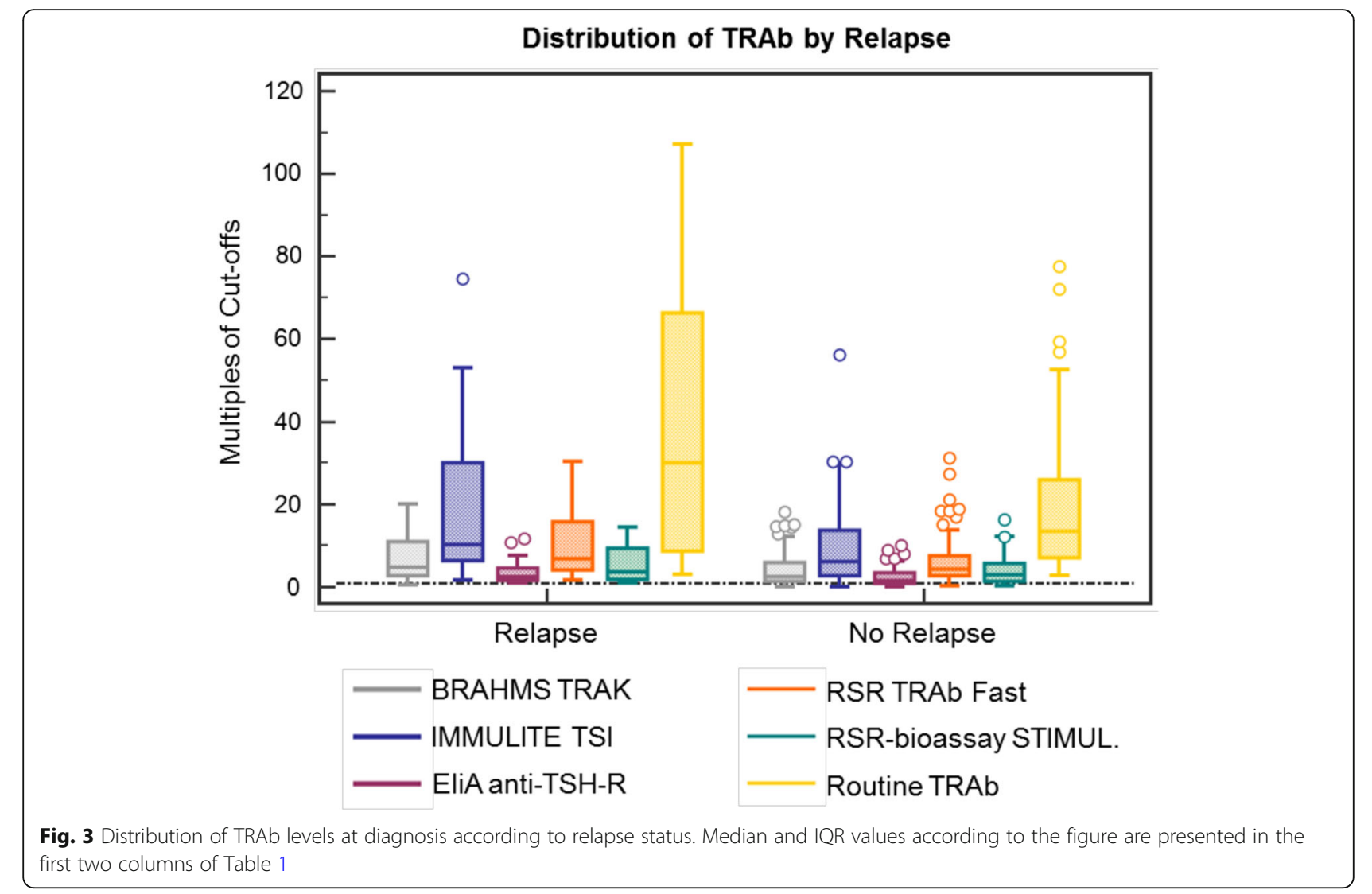

Accordingly, the addition of the new assays to the GREAT score is better than the predictive power of the assays alone. This also explains why previous attempts to predict relapse risk have failed [4, 7, 11-17]. Additionally, it leaves ample space for further research, either on even more specific TRAb or entirely new biomarkers (e.g. cytokines, genetic markers).

We acknowledge several limitations in our study. First, this study is retrospective in design. However, we could gather most data from medical records and we have a long enough follow-up. Second, although we analyzed the blood samples of 332 patients, we had to exclude all but 83 from analysis because a lot of samples were drawn long after ATD treatment initiation. As an exclusion criterion, we chose an ongoing ATD therapy duration for more than 2.5 months. We randomly chose this cut-off as it allowed us to use approximately $1 / 3$ of our dataset. Although, there is a steady fall in TRAb levels during ATD treatment, we do not think that this has inflicted our results. Whereas TRAb levels seem to fall more strongly within 1-3 months after thyroidectomy [29], this decline is less pronounced in patients receiving ATD therapy [30-32]. Thus, we think that including blood samples from patients being up to 2.5 months under ATD therapy did not introduce substantial bias.

Third, we have longer treatment times than recommended by current evidence [4,33]. Median treatment time was similar in both groups ( 19 vs. 18 months). This is explained by our retrospective design. Physicians and

Table 4 Hazard ratios for relapse fitted with new TRAb's 4th versus 1st-3rd quartile and into GREAT ${ }^{a}$

\begin{tabular}{llllll}
\hline Assay & $\begin{array}{l}\text { Recommended cut-offs by the } \\
\text { manufacturer }\end{array}$ & $\begin{array}{l}\text { Level of } \\
\mathrm{Q}_{4}\end{array}$ & $\begin{array}{l}\mathrm{HR} \text { for assay alone } \\
\left(\mathrm{Q}_{4} \text { vs. } \mathrm{Q}_{1-3}\right)(95 \% \mathrm{Cl})\end{array}$ & $\begin{array}{l}\text { HR for GREAT Class II } \\
\text { with new assay }(95 \% \mathrm{Cl})\end{array}$ & $\begin{array}{l}\text { HR for GREAT Class III } \\
\text { with new assay }(95 \% \mathrm{Cl})\end{array}$ \\
\hline BRAHMS TRAK & $\geq 1.80 \mathrm{U} / \mathrm{L}$ & $\geq 8.10 \mathrm{U} / \mathrm{L}$ & $3.53(1.35-9.22)$ & $2.02(0.64-6.36)$ & $3.11(0.57-17.07)$ \\
IMMULITE TSI & $\geq 0.55 \mathrm{U} / \mathrm{L}$ & $\geq 5.66 \mathrm{U} / \mathrm{L}$ & $3.12(1.20-8.12)$ & $3.73(0.84-16.44)$ & $3.01(0.27-33.34)$ \\
EliA anti-TSH-R & $\geq 2.90 \mathrm{U} / \mathrm{L}$ & $\geq 7.40 \mathrm{U} / \mathrm{L}$ & $4.52(1.71-11.99)$ & $2.44(0.79-7.60)$ & $2.37(0.26-21.18)$ \\
RSR TRAb Fast & $\geq 1.00 \mathrm{U} / \mathrm{L}$ & $\geq 7.21 \mathrm{U} / \mathrm{L}$ & $4.41(1.66-11.71)$ & $2.47(0.80-7.72)$ & $1.96(0.22-17.62)$ \\
RSR-bioassay & $\geq 150 \%$ & $\geq 711 \%$ & $3.63(1.39-9.46)$ & $\mathrm{N} / \mathrm{A}$ & $\mathrm{N} / \mathrm{A}$ \\
STIMULATION & & & & &
\end{tabular}




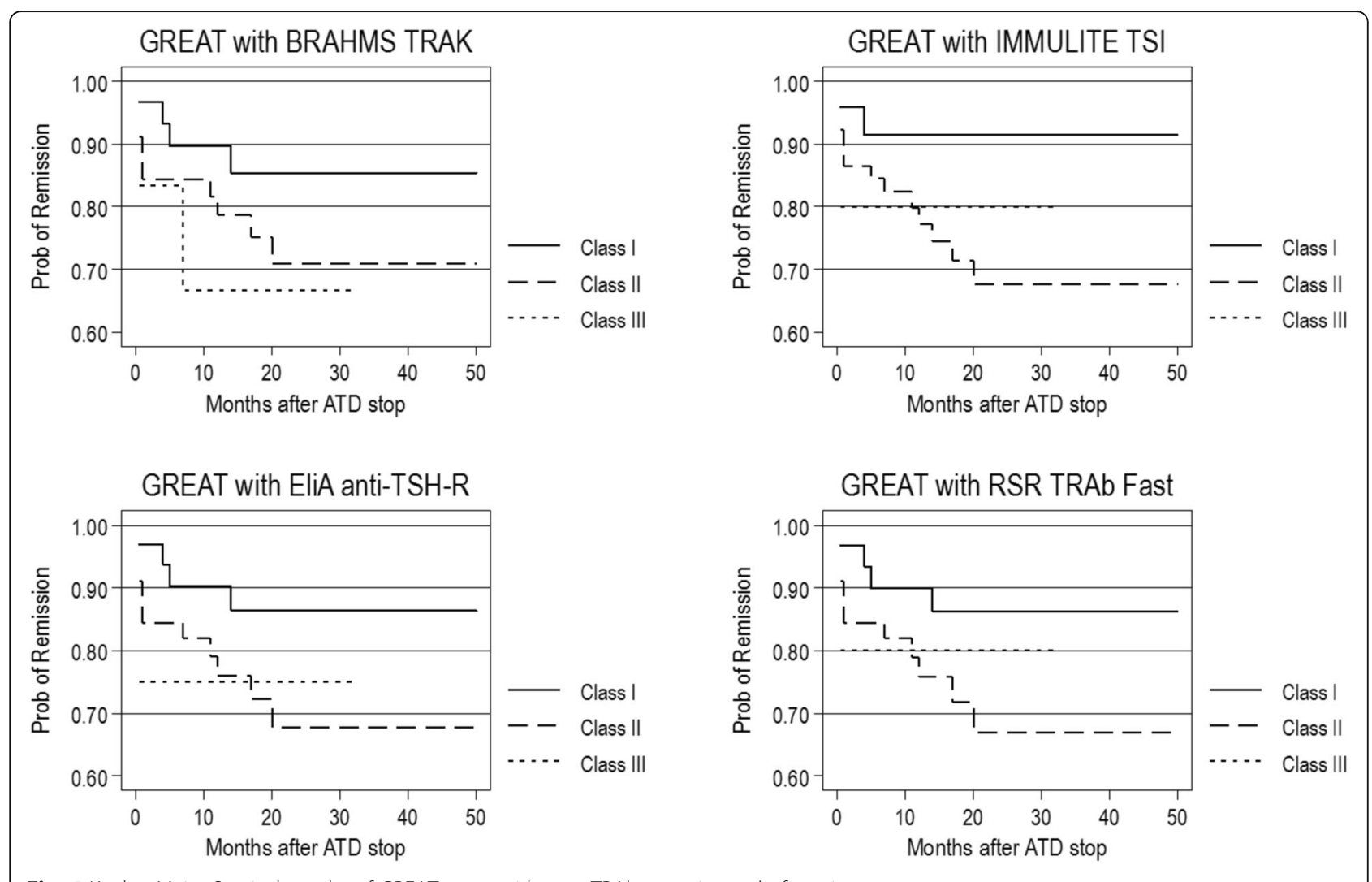

Fig. 4 Kaplan-Meier-Survival graphs of GREAT score with new TRAb assay instead of routine assay

patients usually opt for an extended medical therapy before referral to a thyroid ablative procedure. We hold it unlikely that this might have influenced the results, as treatment duration over 18 months have been found to be of no benefit regarding relapse rate [4].

Forth, our study centers used different routine TRAb assays over the time course of our study. One might argue, that this might have introduce bias. In this case, it should be expected that our results were shifted towards non-significant findings as it disperses our baseline values. Nevertheless, we still found good prognostic accuracy despite inconsistencies in our data set compared to the one from the original GREAT score publication [5], underscoring the consistency of the GREAT score.

Fifth, we used a convenience sample based on a biological repository and had only limited samples available for measurement of TRAbs. Also, we did not use the novel Thyretain bioassay which may have much better performance compared to older bioassays [34]. This should be evaluated in future studies.

Finally, due to our inclusion criteria, seronegative patients with Graves' hyperthyroidism are not represented in our study and it remains unclear how well our findings apply to this patient population. However, every new TRAb assay generation into clinical practice has reduced this population further [19]. It is believed that even those seronegative have TRAb production confined to the thyroid itself or adjacent lymph nodes [35].

\section{Conclusions}

Based on this retrospective analysis, all the studied TRAb assays, but not the bioassay, seem to have better diagnostic and predictive abilities. Thus, they improve assessment of diagnosis and relapse risk in GD, which influences initial treatment decisions. Due to the small sample size and retrospective design with possible selection bias, our data need prospective validation.

\section{Additional file}

Additional file 1: Supplementary information on the assays tested. Table S1. Specifications of routine assays used. Figure S1. Patient inclusion diagram. Figure S2. ROC graphs of TRAb assays with routine assay as reference. Figure S3. Kaplan-Meier-Survival graphs of TRAb assays fourth versus first to third quartile. (DOCX $373 \mathrm{~kb}$ )

\section{Abbreviations}

ATD: Antithyroid drugs; AUC: Area under the receiver operator curve; GD: Graves' disease; GREAT: Graves' Recurrent Events After Therapy; HR: Hazard ratio; IQR: Interquartile range; ROC: Anti-thyroperoxidase; T4 and T3: Thyroxine and triiodothyronine; TPO-Ab: Antibodies; TRAb: TSH-receptor antibody tests; TRAb: TSH-receptor autoantibodies; TSH: Thyrotropin

Acknowledgments

Not applicable. 


\section{Funding}

This study was supported in part by the Swiss National Science Foundation (SNSF Professorship, PP00P3_150531 / 1) and the Research Council of the Kantonsspital Aarau (1410.000.044). Funders had no role in the design, analysis or writing of this article. Commercial companies provided the testing reagents only (TSI Assay, Siemens; EliA anti-TSH-R, Thermo Fisher; TRAK human KRYPTOR, B.R.A.H.M.S Thermo Fisher; TRAb Fast ELISA, RSR). TSH receptor stimulating/ blocking activities were assessed by bioassays at RSR facility (Cardiff, UK). The Sponsors do have no influence on study planning or publication.

\section{Availability of data and materials}

The datasets analyzed during the current study are not publicly available due to restrictions imposed by Swiss law. They might be available from the corresponding author on reasonable request and if legal implications are fulfilled.

\section{Authors' contributions}

$\mathrm{RJ}, \mathrm{NI}, \mathrm{EM}$ and TS analyzed data and wrote the first draft of the manuscript with primary responsibility for the final content. $\mathrm{RJ}, \mathrm{NI}, \mathrm{MK}, \mathrm{FB}$, and $\mathrm{AK}$ retrieved clinical data from medical records. LB, PS and BM initiated the study and provided intellectual support. AH, EM, and LB performed laboratory measurements. CM and MK handled and stored blood samples. All authors read and approved the final manuscript.

\section{Ethics approval and consent to participate}

All procedures performed in studies involving human participants were in accordance with the ethical standards of the institutional and/or national research committee and with the 1964 Helsinki declaration and its later amendments or comparable ethical standards. The study protocol was approved by the local ethics committee (Ethikkommission Nordwest- und Zentralschweiz (EKNZ) Project No. 2015/227). Need for informed consent was waived by the local ethics committee due retrospective nature of analysis with no impact on health outcome.

\section{Consent for publication}

Not applicable.

\section{Competing interests}

BM and PS received research support by Thermo Fisher Scientific, Roche Diagnostics, Abbott and Siemens unrelated to this study. All authors confirm that they do not have a conflict of interest associated with this manuscript.

\section{Publisher's Note}

Springer Nature remains neutral with regard to jurisdictional claims in published maps and institutional affiliations.

\section{Author details}

${ }^{1}$ Medical University Department, Clinic for Endocrinology, Diabetes \& Metabolism, Kantonsspital Aarau, Tellstrasse, CH-5001 Aarau, Switzerland. ${ }^{2}$ Department of Laboratory Medicine, Kantonsspital Aarau, Aarau, Switzerland. ${ }^{3}$ Medical Faculty of the University of Basel, Basel, Switzerland. ${ }^{4}$ Endonet, Basel, Switzerland.

\section{Received: 4 January 2019 Accepted: 1 April 2019}

Published online: 25 April 2019

\section{References}

1. Brent GA. Clinical practice. Graves' disease. N Engl J Med. 2008;358(24):2594605.

2. Franklyn JA, Boelaert K. Thyrotoxicosis. Lancet. 2012;379(9821):1155-66.

3. Kamijo K, Murayama H, Uzu T, Togashi K, Olivo PD, Kahaly GJ. Similar clinical performance of a novel chimeric thyroid-stimulating hormone receptor bioassay and an automated thyroid-stimulating hormone receptor binding assay in Graves' disease. Thyroid. 2011;21(12):1295-9.

4. Abraham P, Avenell A, McGeoch SC, Clark LF, Bevan JS. Antithyroid drug regimen for treating Graves' hyperthyroidism. Cochrane Database Syst Rev. 2010;(1):CD003420. https://doi.org/10.1002/14651858.CD003420.pub4.

5. Vos XG, Endert E, Zwinderman AH, Tijssen JG, Wiersinga WM. Predicting the risk of recurrence before the start of Antithyroid drug therapy in patients with Graves' hyperthyroidism. J Clin Endocrinol Metab. 2016;101(4):1381-9.

6. Struja T, Kaeslin M, Boesiger F, Jutzi R, Imahorn N, Kutz A, et al. External validation of the GREAT score to predict relapse risk in Graves' disease: results from a multicenter, retrospective study with 741 patients. Eur Endocrinol. 2017;176(4):413-9.

7. Struja T, Fehlberg H, Kutz A, Guebelin L, Degen C, Mueller B, et al. Can we predict relapse in Graves' disease? Results from a systematic review and meta-analysis. Eur J Endocrinol. 2017;176(1):87-97.

8. Allelein S, Ehlers M, Goretzki S, Hermsen D, Feldkamp J, Haase $M$, et al. Clinical evaluation of the first automated assay for the detection of stimulating TSH receptor autoantibodies. Horm Metab Res. 2016;48(12): 795-801

9. Frank CU, Braeth S, Dietrich JW, Wanjura D, Loos U. Bridge technology with TSH receptor chimera for sensitive direct detection of TSH receptor antibodies causing Graves' disease: analytical and clinical evaluation. Horm Metab Res. 2015;47(12):880-8.

10. Tozzoli R, D'Aurizio F, Villalta D, Giovanella L. Evaluation of the first fully automated immunoassay method for the measurement of stimulating TSH receptor autoantibodies in Graves' disease. Clin Chem Lab Med. 2017;55(1): 58-64.

11. Autilio C, Morelli R, Locantore P, Pontecorvi A, Zuppi C, Carrozza C. Stimulating TSH receptor autoantibodies immunoassay: analytical evaluation and clinical performance in Graves' disease. Ann Clin Biochem. 2018;55(1): $172-7$.

12. Diana T, Wuster C, Kanitz M, Kahaly GJ. Highly variable sensitivity of five binding and two bio-assays for TSH-receptor antibodies. J Endocrinol Investig. 2016;39(10):1159-65

13. Luther T, Sjölander S, Kast M, Konrad C. In: Inc. TFS, editor. White paper: EliA anti-TSH-R assay. Karlsruhe: Thermo Fisher Scientic Inc; 2016. p. 1-8.

14. Lytton SD, Li Y, Olivo PD, Kohn LD, Kahaly GJ. Novel chimeric thyroidstimulating hormone-receptor bioassay for thyroid-stimulating immunoglobulins. Clin Exp Immunol. 2010;162(3):438-46.

15. Diana T, Krause J, Olivo PD, Konig J, Kanitz M, Decallonne B, et al. Prevalence and clinical relevance of thyroid stimulating hormone receptorblocking antibodies in autoimmune thyroid disease. Clin Exp Immunol. 2017;189(3):304-9.

16. Smith BR, Bolton J, Young S, Collyer A, Weeden A, Bradbury J, et al. A new assay for thyrotropin receptor autoantibodies. Thyroid. 2004;14(10):830-5.

17. Doroudian S, Pedersen IB, Knudsen CS, Handberg A, Andersen SL. Comparison of three competitive immunoassays for measurement of TSH receptor antibodies in patients with Graves' disease. Scand J Clin Lab Invest. 2017;77(7):535-40

18. Theodoraki A, Jones G, Parker J, Woolman E, Martin N, Perera S, et al. Performance of a third-generation TSH-receptor antibody in a UK clinic. Clin Endocrinol. 2011;75(1):127-33.

19. Tozzoli R, Bagnasco M, Giavarina D, Bizzaro N. TSH receptor autoantibody immunoassay in patients with Graves' disease: improvement of diagnostic accuracy over different generations of methods. Systematic review and meta-analysis. Autoimmun Rev. 2012;12(2):107-13.

20. Hussain YS, Hookham JC, Allahabadia A, Balasubramanian SP. Epidemiology, management and outcomes of Graves' disease-real life data. Endocrine. 2017:56(3):568-78.

21. Laurberg $P$, Wallin $G$, Tallstedt $L$, Abraham-Nordling M, Lundell $G$, Torring $O$. TSH-receptor autoimmunity in Graves' disease after therapy with antithyroid drugs, surgery, or radioiodine: a 5-year prospective randomized study. Eur J Endocrinol. 2008;158(1):69-75.

22. Ilicki A, Gamstedt A, Karlsson FA. Hyperthyroid Graves' disease without detectable thyrotropin receptor antibodies. J Clin Endocrinol Metab. 1992; 74(5):1090-4.

23. Kawai K, Tamai H, Matsubayashi S, Mukuta T, Morita T, Kubo C, et al. A study of untreated Graves' patients with undetectable TSH binding inhibitor immunoglobulins and the effect of anti-thyroid drugs. Clin Endocrinol. 1995; 43(5):551-6.

24. Kahaly GJ, Diana T, Glang J, Kanitz M, Pitz S, Konig J. Thyroid stimulating antibodies are highly prevalent in Hashimoto's thyroiditis and associated Orbitopathy. J Clin Endocrinol Metab. 2016;101(5): 1998-2004.

25. Wall JR, Lahooti H, El Kochairi I, Lytton SD, Champion B. Thyroid-stimulating immunoglobulins as measured in a reporter bioassay are not detected in patients with Hashimoto's thyroiditis and ophthalmopathy or isolated upper eyelid retraction. Clin Ophthalmol. 2014;8:2071-6.

26. Diana T, Li Y, Olivo PD, Lackner KJ, Kim H, Kanitz M, et al. Analytical performance and validation of a bioassay for thyroid-blocking antibodies. Thyroid. 2016;26(5):734-40. 
27. Lytton SD, Kahaly GJ. Bioassays for TSH-receptor autoantibodies: an update. Autoimmun Rev. 2010;10(2):116-22.

28. Giuliani C, Saji M, Bucci I, Napolitano G. Bioassays for TSH receptor autoantibodies, from FRTL-5 cells to TSH receptor-LH/CG receptor chimeras: the contribution of Leonard D. Kohn. Front Endocrinol (Lausanne). 2016;7:103.

29. Yoshioka W, Miyauchi A, Ito M, Kudo T, Tamai H, Nishihara E, et al. Kinetic analyses of changes in serum TSH receptor antibody values after total thyroidectomy in patients with Graves' disease. Endocr J. 2016;63(2):179-85.

30. Michelangeli V, Poon C, Taft J, Newnham H, Topliss D, Colman P. The prognostic value of thyrotropin receptor antibody measurement in the early stages of treatment of Graves' disease with antithyroid drugs. Thyroid. 1998; 8(2):119-24

31. Takasu N. Remission of Graves' hyperthyroidism predicted by smooth decreases of thyroid-stimulating antibody and thyrotropin-binding inhibitor immunoglobulin during antithyroid drug treatment. Thyroid. 2000;10(10):891-6.

32. Eckstein AK, Plicht M, Lax H, Neuhauser M, Mann K, Lederbogen S, et al. Thyrotropin receptor autoantibodies are independent risk factors for Graves' ophthalmopathy and help to predict severity and outcome of the disease. J Clin Endocrinol Metab. 2006;91(9):3464-70.

33. Bartalena L, Burch HB, Burman KD, Kahaly GJ. A 2013 European survey of clinical practice patterns in the management of Graves' disease. Clin Endocrinol. 2016;84(1):115-20.

34. Giuliani C, Cerrone D, Harii N, Thornton M, Kohn LD, Dagia NM, et al. A TSHR-LH/CGR chimera that measures functional thyroid-stimulating autoantibodies (TSAb) can predict remission or recurrence in Graves' patients undergoing antithyroid drug (ATD) treatment. J Clin Endocrinol Metab. 2012;97(7):E1080-7.

35. Orgiazzi J. Thyroid autoimmunity. Presse Med. 2012;41(12 P 2):e611-25.

Ready to submit your research? Choose BMC and benefit from:

- fast, convenient online submission

- thorough peer review by experienced researchers in your field

- rapid publication on acceptance

- support for research data, including large and complex data types

- gold Open Access which fosters wider collaboration and increased citations

- maximum visibility for your research: over $100 \mathrm{M}$ website views per year

At $\mathrm{BMC}$, research is always in progress.

Learn more biomedcentral.com/submissions 